\title{
Treatment of Triple-Negative Breast Cancer Using Anti- EGFR-Directed Radioimmunotherapy Combined with Radiosensitizing Chemotherapy and PARP Inhibitor
}

\author{
Fares Al-Ejeh ${ }^{1}$, Wei Shi ${ }^{1}$, Mariska Miranda ${ }^{1}$, Peter T. Simpson ${ }^{2}$, Ana Cristina Vargas ${ }^{2}$, Sarah Song ${ }^{2,3}$, \\ Adrian P. Wiegmans ${ }^{1}$, Alex Swarbrick ${ }^{4,5}$, Alana L. Welm ${ }^{6}$, Michael P. Brown ${ }^{7,8}$, Georgia Chenevix-Trench ${ }^{9}$, \\ Sunil R. Lakhani ${ }^{2,10,11}$, and Kum Kum Khanna ${ }^{1}$ \\ ${ }^{1}$ Signal Transduction Laboratory, Queensland Institute of Medical Research, Brisbane, Queensland, Australia; ${ }^{2}$ UQ Centre for \\ Clinical Research, University of Queensland, Brisbane, Queensland, Australia; ${ }^{3}$ Queensland Centre for Medical Genomics, Institute \\ for Molecular Bioscience, University of Queensland, St. Lucia, Queensland, Australia; ${ }^{4}$ St. Vincent's Clinical School, Faculty of \\ Medicine, University of New South Wales, Darlinghurst, New South Wales, Australia, ${ }^{5}$ Cancer Research Program, Garvan Institute of \\ Medical Research, Darlinghurst, New South Wales, Australia; ${ }^{6}$ Department of Oncological Sciences, Huntsman Cancer Institute, \\ University of Utah, Salt Lake City, Utah; ${ }^{7}$ Cancer Clinical Trials Unit, Royal Adelaide Hospital Cancer Centre, Adelaide, South \\ Australia, Australia; ${ }^{8}$ School of Medicine, University of Adelaide, Adelaide, South Australia, Australia; ${ }^{9}$ Cancer Genetics Laboratory, \\ Queensland Institute of Medical Research, Brisbane, Queensland, Australia; ${ }^{10}$ School of Medicine, University of Queensland, Brisbane, \\ Queensland, Australia; and ${ }^{11}$ Pathology Queensland, Royal Brisbane and Women's Hospital, Brisbane, Queensland, Australia
}

Triple-negative breast cancer (TNBC) is associated with poor survival. Chemotherapy is the only standard treatment for TNBC. The prevalence of BRCA1 inactivation in TNBC has rationalized clinical trials of poly(adenosine diphosphate ribose) polymerase (PARP) inhibitors. Similarly, the overexpression of epidermal growth factor receptor (EGFR) rationalized anti-EGFR therapies in this disease. However, clinical trials using these 2 strategies have not reached their promise. In this study, we used EGFR as a target for radioimmunotherapy and hypothesized that EGFR-directed radioimmunotherapy can deliver a continuous lethal radiation dose to residual tumors that are radiosensitized by PARP inhibitors and chemotherapy. Methods: We analyzed EGFR messenger RNA in published gene expression array studies and investigated EGFR protein expression by immunohistochemistry in a cohort of breast cancer patients to confirm EGFR as a target in TNBC. Preclinically, using orthotopic and metastatic xenograft models of EGFR-positive TNBC, we investigated the effect of the novel combination of ${ }^{177} \mathrm{Lu}$ labeled anti-EGFR monoclonal antibody, chemotherapy, and PARP inhibitors on cell death and the survival of breast cancer stem cells. Results: In this first preclinical study of anti-EGFR radioimmunotherapy in breast cancer, we found that anti-EGFR radioimmunotherapy is safe and that TNBC orthotopic tumors and established metastases were eradicated in mice treated with anti-EGFR radioimmunotherapy combined with chemotherapy and PARP inhibitors. We showed that the superior response to this triple-agent combination therapy was associated with apoptosis and eradication of putative breast cancer stem cells. Conclusion: Our data support further preclinical investi-

\footnotetext{
Received Jul. 19, 2012; revision accepted Dec. 27, 2012.

For correspondence contact: Fares Al-Ejeh, Queensland Institute of Medical

Research, Level 9, 300 Herston Rd., Brisbane, QLD 4006, Australia.

E-mail: fares.al-ejeh@qimr.edu.au

Published online Apr. 5, 2013.

COPYRIGHT (C) 2013 by the Society of Nuclear Medicine and Molecular Imaging, Inc.
}

gations toward the development of combination therapies using systemic anti-EGFR radioimmunotherapy for the treatment of recurrent and metastatic TNBC.

Key Words: triple-negative breast cancer; EGFR; PARP inhibitor; radioimmunotherapy; combination therapy

J Nucl Med 2013; 54:913-921

DOI: 10.2967/jnumed.112.111534

B reast cancer is the leading cause of cancer-related mortality among women worldwide, and 30\%-40\% of breast cancer patients will develop metastatic disease. Currently, no targeted therapies are used as the standard of care for triple-negative breast cancer (TNBC), which is defined by the lack of expression of human epidermal growth factor receptor 2 (HER2), estrogen receptor, and progesterone receptor. This aggressive disease, relative to other breast cancer subtypes, is associated with a higher incidence of recurrence and distant metastasis and a poorer survival (1). Conventional cytotoxic chemotherapy remains the only treatment option for TNBC.

In 2 large studies of breast cancer patients $(2,3), 17 \%-24 \%$ of patients were identified as having TNBC by immunohistochemistry. Triple-negative status still identifies a heterogeneous group with significant differences in molecular markers and survival. The basal-like subtype of TNBC, which stains positively for high-molecular-weight cytokeratins or epidermal growth factor receptor (EGFR) by immunohistochemistry, represents $53 \%-67 \%$ of TNBC (2-4). Interestingly, although basal-like TNBC has the poorest outcome $(3,4)$, patients with these tumors have a disease-free survival benefit with adjuvant chemotherapy $(2,4)$. 
Several studies have reported high expression of EGFR by immunohistochemistry in $40 \%-70 \%$ of TNBC patients $(2,4-6)$, with high expression correlating with poor outcome and decreased disease-free survival (5). Elevation of EGFR messenger RNA (mRNA), as well as activation of the EGFR pathway in gene expression microarray studies, is a characteristic of basal-like breast cancer $(2,7)$ and is associated with poor prognostic signatures in almost all basal-like tumors (8). Thus, EGFR represents a clinically relevant target in basal-like TNBC. Another target for TNBC is poly(adenosine diphosphate ribose) polymerase-1 (PARP1). The high prevalence of inactivation of BRCA1, which is involved in the homologous recombination DNA repair pathway, in TNBC supports combining chemotherapy or radiotherapy with PARP inhibitors. A synthetic lethality approach using PARP inhibition has been the subject of recent reviews (9).

Although both EGFR and PARP may be attractive targets for breast cancer drugs, EGFR inhibition (antibodies or small inhibitors) or PARP inhibition has shown limited success in treating TNBC (1). In this study, we used EGFR as a target for radioimmunotherapy in EGFR-positive TNBC. We hypothesized that anti-EGFR monoclonal antibody $(\mathrm{mAb})$ labeled with the therapeutic radionuclide ${ }^{177} \mathrm{Lu}$ would deliver a lethal and continuous radiation dose to radiosensitized tumor cells remaining after chemical tumor debulking and PARP inhibition.

\section{MATERIALS AND METHODS}

\section{EGFR Gene and Protein Expression in Breast Cancer Patients}

Gene expression array data from 5 studies (GSE12276, GSE21653, GSE2990, GSE3494, and GSE2034) were obtained from the Gene Expression Omnibus. The datasets were analyzed for EGFR mRNA expression across the intrinsic subtypes as described in Supplemental Figure 4 (supplemental materials are available online only at http://jnm.snmjournals.org).

For immunohistochemical analysis of EGFR, approval was obtained from the Human Research Ethics Committee of the Royal Brisbane and Women's Hospital and the University of Queensland to use human breast tumor samples for research. Tissue microarrays were enriched for high-grade invasive ductal carcinomas for which estrogen receptor, progesterone receptor, and HER2 immunohistochemical data were also available. Standard immunohistochemistry was performed on tissue microarray sections to evaluate EGFR protein expression (Supplemental Methods).

\section{Orthotopic and Metastatic Breast Xenograft Models}

Cell culture conditions and the production and radiolabeling of the anti-EGFR mouse mAb (clone 225), precursor to the derived chimeric human:murine antibody $\mathrm{C} 225$ commercially known as cetuximab, are detailed in the Supplemental Methods. The Animal Ethics Committee of the Queensland Institute of Medical Research gave approval for use of the mice. We used 2 orthotopic mouse models using the TNBC MDA-MB-231 cell line and the patient-derived TNBC xenograft HCI-002. We also used an experimental metastasis model established from intravenous injection of the MDA-MB-231 cell line (Supplemental Methods).
For the orthotopic mammary fat pad model, treatments were initiated when tumors were $50 \pm 1 \mathrm{~mm}^{3}$ as calculated by caliper measurement of the longest $(a)$ and shortest $(b)$ diameters and the following equation: tumor volume $\left(\mathrm{mm}^{3}\right)=a / 2 \times b^{2}$. For the metastasis model, treatments were initiated $14 \mathrm{~d}$ after inoculation, when metastases were established. Treatment groups consisted of 5 mice each for the MDA-MB-231 orthotopic model (3 independent experiments were performed), 10 mice each for the patientderived HCI-002 orthotopic model, and 5-7 mice each in the MDA-MB-231 established metastasis model (2 independent experiments were performed). Docetaxel, doxorubicin, and the PARP inhibitor rucaparib (Pfizer's PF-01367338) were purchased from Selleck Chemicals LLC and diluted in a 5\% solution of D-glucose in phosphate-buffered saline for intraperitoneal injection. Docetaxel and doxorubicin were injected as single doses, whereas PARP inhibitor was injected as 5 daily doses. For radioimmunotherapy, ${ }^{177} \mathrm{Lu}$-anti-EGFR $\mathrm{mAb}$ in phosphate-buffered saline was injected in the tail vein $24 \mathrm{~h}$ after chemotherapy. Control mice received vehicle solutions, and EGFR control mice were injected with unlabeled anti-EGFR mAb. Mouse weights and tumor volumes were measured twice per week in the first $3 \mathrm{wk}$ of treatment and then once per week.

\section{Cell Death Assays in In Vivo and Ex Vivo Analyses}

Activation of caspase-3 in vivo was detected by live animal imaging on day 7 after treatments using the VivoGlo Caspase-3/7 substrate (Promega Corp.). Images were acquired $10 \mathrm{~min}$ after intraperitoneal injection of the substrate $(25 \mathrm{mg} / \mathrm{kg})$.

Standard immunoblotting was performed using lysates prepared from freshly isolated tumors from mice $2 \mathrm{~d}$ after administration of ${ }^{177} \mathrm{Lu}$-anti-EGFR mAb. Membranes were probed with antibodies against phosphorylated DNA-dependent protein kinase (DNA-PK) at serine 2056 (Abcam), DNA-PK, RAD51 (Santa Cruz Biotech), and $\beta$-actin (Sigma-Aldrich) and developed using Chemilucent Plus (Millipore).

Additional apoptosis and cell death assays were performed ex vivo on tumors 3 and $7 \mathrm{~d}$ after treatments. Single-cell suspensions were prepared by collagenase $\mathrm{B}$ digestion as described previously (10) for annexin V/7-AAD apoptosis assays and DNA-content cell

TABLE 1

Immunohistochemistry Scores for EGFR Staining in Cohort of Breast Cancer

\begin{tabular}{|c|c|c|c|c|}
\hline Variable & All & $\mathrm{EGFR}^{+}$ & EGFR $^{-}$ & $P^{*}$ \\
\hline Grade & 106 & $22(21 \%)$ & 84 (79\%) & \\
\hline 1 & 3 & $0(0 \%)$ & $3(100 \%)$ & \\
\hline 2 & 22 & $4(18 \%)$ & $18(82 \%)$ & \\
\hline 3 & 80 & $17(21 \%)$ & 63 (79\%) & \\
\hline Not applicable $^{\dagger}$ & 1 & 1 & 0 & \\
\hline TNBC status & & & & 0.005 \\
\hline TNBC & 28 & $11(39 \%)$ & 17 (61\%) & \\
\hline Non-TNBC & 78 & $11(14 \%)$ & 67 (86\%) & \\
\hline Non-TNBC & & & & 0.029 \\
\hline 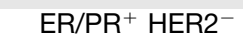 & 23 & $1(4 \%)$ & 22 (96\%) & \\
\hline $\mathrm{HER}^{+}{ }^{+}$ & 55 & $10(18 \%)$ & $45(82 \%)$ & \\
\hline
\end{tabular}

${ }^{\star} \chi^{2}$ test.

${ }^{\dagger}$ Grade not available for 1 case.

$\mathrm{ER} / \mathrm{PR}=$ estrogen receptor/progesterone receptor.

Data are $N$, followed by percentage in parentheses. 
cycle assays (Invitrogen, Life Technologies) as per manufacturer instructions and analyzed using a FACSCalibur flow cytometer (BD Biosciences). Formalin-fixed, paraffin-embedded tumors were used to prepare 7- $\mu \mathrm{m}$ tissue sections for hematoxylin and eosin staining or immunohistochemistry with rabbit IgG against cleaved PARP1 (1:100 of stock; Promega) as described previously (10). Slides were scanned using ScanScope TX (Aperio Technologies).

The percentage of breast cancer stem cells (BCSCs) in vitro was investigated using a previously published method (11). Briefly, the cells were treated with single agents or combinations as described in the Supplemental Methods, and after 2 rounds of 3-d treatment, the cells were collected and stained $\left(30 \mathrm{~min}\right.$ at $\left.4^{\circ} \mathrm{C}\right)$ with anti-CD44 PE-Cy5-mAb, anti-CD24 PE-mAb, and anti-EpCAM FITC-mAb (all from BD Biosciences) as per manufacturer instructions. In vivo, the tumors were excised after treatments to prepare single-cell suspensions as described earlier. Single-cell suspensions were stained with CD44, CD24, and EpCAM antibodies before analysis using the FACSCalibur flow cytometer.

Statistical analysis was performed with Prism, version 5.0 (GraphPad Software). The types of tests used are stated in the figure legends.

\section{RESULTS}

\section{EGFR mRNA and Protein in Basal-Like TNBC}

We found that EGFR mRNA levels are significantly higher in basal-like breast cancer than in luminal A and B and HER2 molecular subtypes but not in normal-like breast cancer (Supplemental Fig. 4). We also used immunohistochemistry on tissue microarrays of a sporadic breast cancer cohort and found that a higher percentage of TNBC $(39 \%)$ than of non-TNBC (14\%) was positive for EGFR $(P=$ 0.005 , Table 1).

\section{Combination Therapy with ${ }^{177}$ Lu-Anti EGFR mAb}

On the basis of toxicity and efficacy studies (Supplemental Results), we determined that chemotherapy with $2 \mathrm{mg}$ of docetaxel and $10 \mathrm{mg}$ of doxorubicin per kilogram and radioimmunotherapy with $300 \mathrm{MBq}$ of ${ }^{177} \mathrm{Lu}$-antiEGFR per kilogram were suitable to investigate with PARP inhibitor. Indeed, as shown in Fig. 1, 5 daily doses of PARP inhibitor at $2 \mathrm{mg} / \mathrm{kg}$ potentiated chemotherapy (tumor

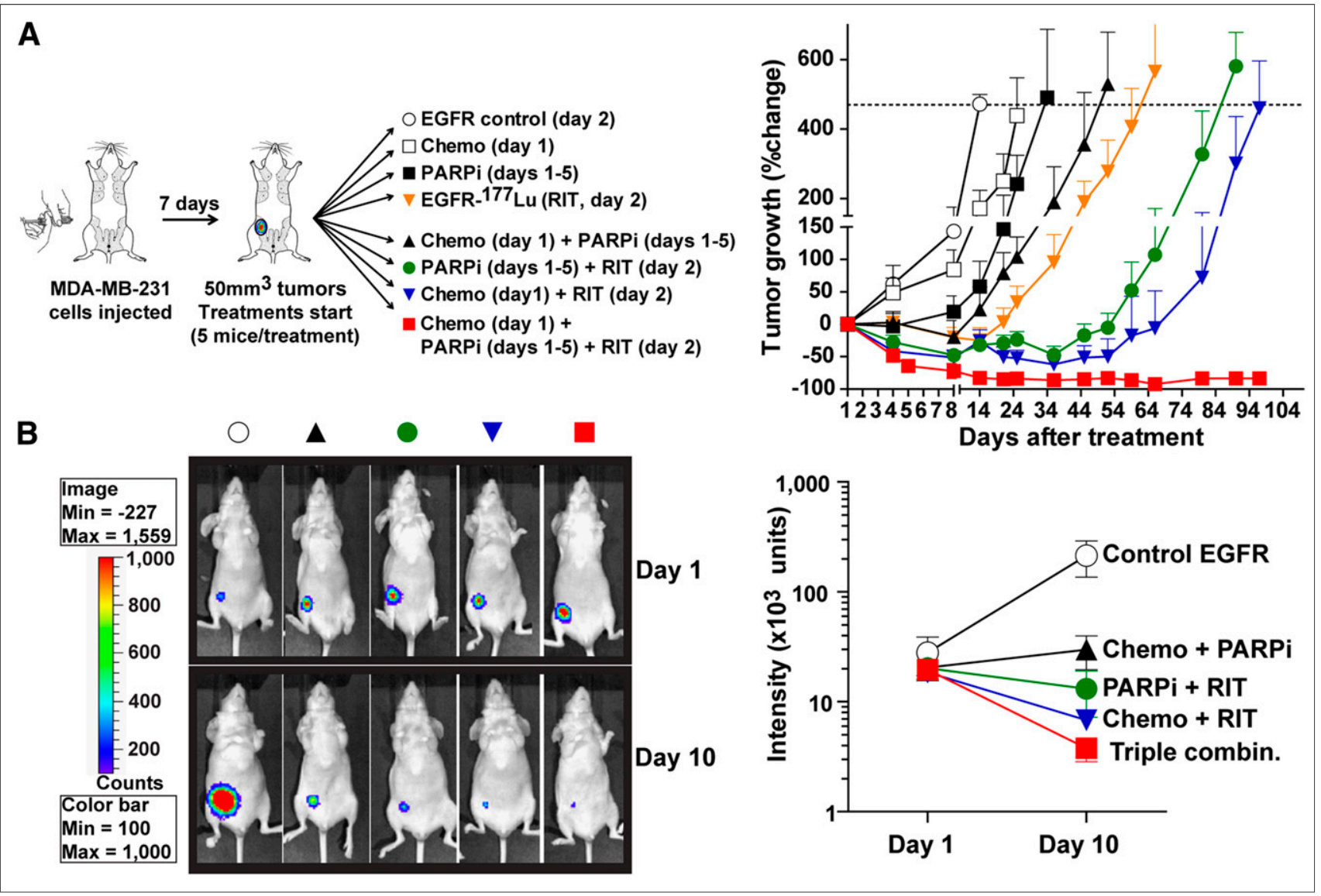

FIGURE 1. Eradication of MDA-MB-231 mammary fat pad xenografts using combination of EGFR-directed radioimmunotherapy with chemotherapy and PARP inhibitor. Female nude BALB/c mice bearing MDA-MB-231 mammary fat pad tumors $(5$ mice per group) either were left untreated as vehicle controls or were treated with unlabeled anti-EGFR mAb (control EGFR), doxorubicin (10 $\mathrm{mg} / \mathrm{kg}$ ) and docetaxel $\left(2 \mathrm{mg} / \mathrm{kg}\right.$ ) chemotherapy (chemo), PARP inhibitor at $2 \mathrm{mg} / \mathrm{kg}$ daily for $5 \mathrm{~d}$ (PARPi), or ${ }^{177}$ Lu-anti-EGFR mAb at $300 \mathrm{MBq} / \mathrm{kg}$ (RIT). Any treatment without radioimmunotherapy included unlabeled anti-EGFR mAb as control. Mouse weights and tumor volumes were monitored. No significant weight loss was observed. (A) Treatment schedules and tumor growth curves. Data are average percentage change $( \pm S E M)$ in tumor volume. (B) Representative bioluminescence images of mice and mean luminescence intensities ( \pm SEM) on days 1 and 10 after treatment (Supplemental Fig. 1 shows images of all mice). 
growth delay, $38 \mathrm{~d}$ for chemotherapy and PARP inhibitor vs. $11 \mathrm{~d}$ for chemotherapy alone) and ${ }^{177} \mathrm{Lu}$-anti-EGFR radioimmunotherapy (tumor growth delay, $76 \mathrm{~d}$ for PARP inhibitor and radioimmunotherapy vs. $52 \mathrm{~d}$ for radioimmunotherapy alone). Chemotherapy potentiated ${ }^{177} \mathrm{Lu}$-antiEGFR radioimmunotherapy (tumor growth delay, $83 \mathrm{~d}$ for chemotherapy and radioimmunotherapy vs. $52 \mathrm{~d}$ for radioimmunotherapy alone). Treatment with naked anti-EGFR $\mathrm{mAb}$ alone, which acted as a control for radioimmunotherapy-containing treatments, had no effect on tumor growth, which was the same as in mice with untreated tumors. Strikingly, the combination of ${ }^{177} \mathrm{Lu}$-anti-EGFR radioimmunotherapy with chemotherapy and PARP inhibitor (triple-agent combination) eradicated the established xenografts, with no recurrences observed up to $120 \mathrm{~d}$ after treatment (Fig. 1). The efficacy of this triple-agent combination was confirmed by bioluminescence imaging, as minimal signal was observed on day 10 (Fig. 1B). From a total of 15 mice treated with the triple-agent combination, only 1 mouse showed residual disease, and it did not progress $(6.7 \%) ; 14$ mice $(93.3 \%)$ were completely tumor-free up to $120 \mathrm{~d}$.

\section{Biologic Effects of the Triple-Agent Combination In Vivo}

Next, we used bioluminescence imaging with caspase-3/7 substrate and found that the triple-agent combination induced significantly higher caspase activation in vivo than did other combinations (Fig. 2).

We excised tumors $2 \mathrm{~d}$ after radioimmunotherapy to quantify the expression of activated DNA-PK (phosphorylated at serine 2056 [DNA-PK S2056]) and RAD51 protein, central players in the DNA double-strand break repair pathways. As shown in Fig. 3A, DNA-PK was activated (S2056 phosphorylation) in tumors treated with chemotherapy and PARP inhibitor; however, this phosphorylation was inhibited when EGFR-directed radioimmunotherapy was added. In the case of RAD51, chemotherapy and PARP inhibitor did not affect the protein level, but RAD51 protein was significantly reduced in tumors from mice treated with the triple-agent combination. These data suggest that the inhibition of central DNA repair proteins contributed to the efficacy of the triple-agent combination. Additional in vitro studies revealed that this combination caused a delay in the repair of double-strand breaks (Supplemental Results).

To determine the consequence of DNA repair inhibition, we further examined tumor responses ex vivo. In agreement with studies of caspase activation in vivo, ex vivo studies showed significantly higher cleavage of PARP1 (an event mediated by active caspase-3) that persisted up to day 7 when mice were treated with the triple-agent combination (Fig. 3B and Supplemental Fig. 6). Flow cytometry analysis showed high levels of persistent cell death after treatment with the triple combination as shown by lack of cell viability staining (Fig. 3C) and sub-G1

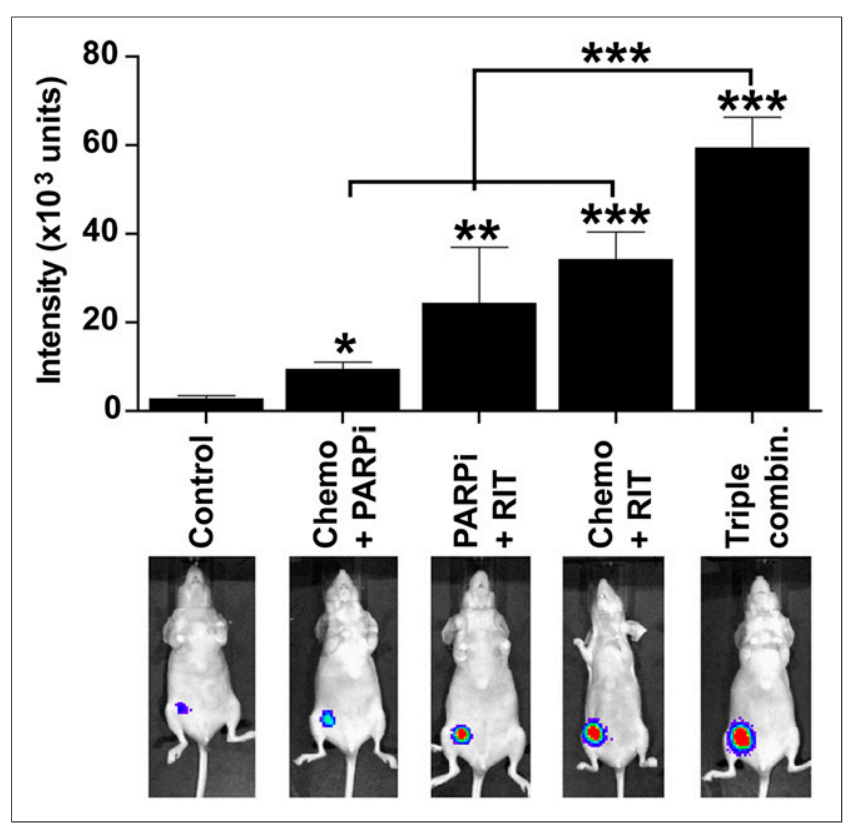

FIGURE 2. In vivo bioluminescent imaging of caspase-3/7 activation after treatment. Female nude BALB/c mice bearing luciferaseexpressing mammary fat pad xenografts of MDA-MB-231 were left untreated or treated as indicated. Treatment doses, schedule, and routes of administration were as described in legend to Figure 1. Seven days after treatment (5 mice per group), mice were injected with VivoGlo caspase $3 / 7$ substrate $(25 \mathrm{mg} / \mathrm{kg}$ ) and imaged $10 \mathrm{~min}$ later. Mean luminescence intensities ( \pm SEM) for caspase- 3 activation are summarized in bar graph. Representative images are shown for each treatment (Supplemental Fig. 2 shows images of all mice). ${ }^{\star} P<0.05,{ }^{\star \star} P<0.01,{ }^{* \star \star} P<0.001$ (1-way ANOVA with Tukey multiple-comparisons posttest).

accumulation (Fig. 3D), corroborating the findings of the apoptosis studies.

BCSCs with the $\mathrm{CD} 44^{+} / \mathrm{CD} 24^{-} / \mathrm{EpCAM}^{+}$profile are enriched by chemotherapeutics in vitro (12). We found that treatment of MDA-MB-231 cells (a TNBC cell line) with unlabeled anti-EGFR mAb did not enrich these cells. The treatment of MDA-MB-231 cells with chemotherapy alone and in combination with PARP inhibitor significantly increased the percentage of BCSCs in vitro (Fig. 4A, left panel). In contrast, treatment with anti-EGFR mAb labeled with ${ }^{177} \mathrm{Lu}$ alone significantly reduced the percentage of $\mathrm{CD}_{4}{ }^{+} / \mathrm{CD} 24^{-} / \mathrm{EpCAM}^{+}$cells, and more so when combined with chemotherapy and PARP inhibitor (Fig. 4A, right panel). In vivo, we found that MDA-MB-231 xenograft tumors remaining on day 10 after treatment with the triple-agent combination contained significantly fewer CD $44^{+} / \mathrm{CD} 24^{-}$cells and more $\mathrm{CD} 44^{-} / \mathrm{CD} 24^{-}$cells than did tumors in control and other treatment arms (Fig. 4B). The loss of CD44 results in defective luminal-myoepithelial cell-cell adhesion and correlates to loss of epithelial-stromal interactions (13) - functions that may affect tumor formation. Importantly and consistently with the in vitro results, the combination of chemotherapy and PARP inhibitor significantly enriched for $\mathrm{CD} 44^{+} / \mathrm{CD} 24^{-} / \mathrm{EpCAM}^{+}$cells in the mammary fat pad xenografts (Fig. 4C). Although the com- 


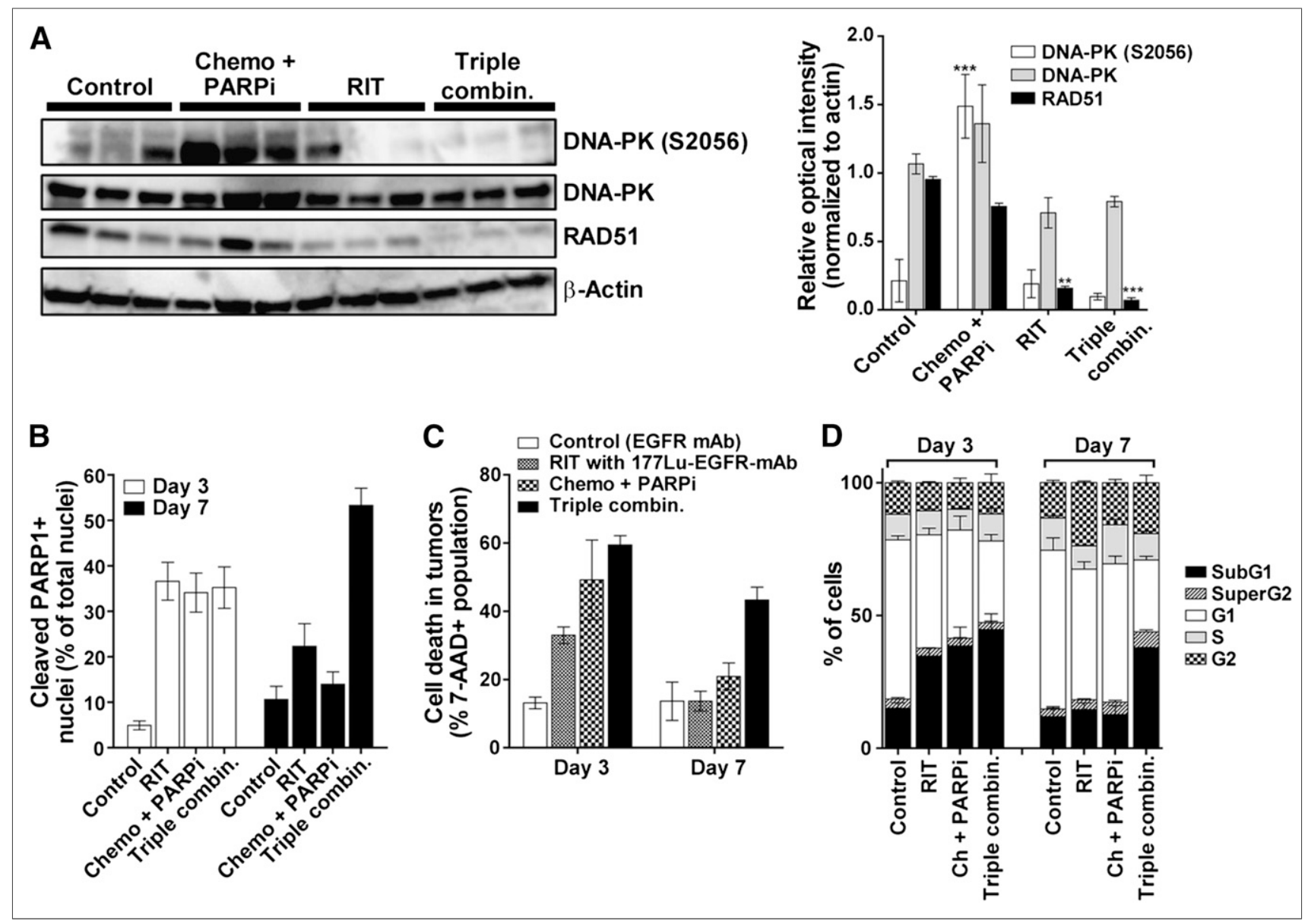

FIGURE 3. Ex vivo investigation of DNA repair proteins and cell death after combination therapy. (A) Immunoblot analysis of tumors excised from mice $2 \mathrm{~d}$ after administration of ${ }^{177} \mathrm{Lu}$-anti-EGFR radioimmunotherapy. Bar graph depicts mean relative intensity of specific bands normalized to loading control $\beta$-actin ( \pm SEM, 3 mice per group). Asterisks denote statistical differences in comparison to tumors from control mice. (B) Quantification of cleaved PARP1 detected by immunohistochemistry of tumors on days 3 and 7 after treatments (Supplemental Fig. 6 presents representative hematoxylin and eosin and cleaved PARP1 immunohistochemistry images). Data are mean ( \pm SEM) percentage of nuclei staining positively for cleaved PARP1 to total number of nuclei (hematoxylin counterstain). (C and D) Quantification of percentage cell death using 7-AAD vital dye $(C)$ and proportion of cells in different cell cycle phases by FACSCalibur analysis (D) in single-cell suspensions prepared from tumors at 3 and $7 \mathrm{~d}$ after treatment $(3$ tumors $/ \mathrm{d} /$ treatment). Ch and chemo $=$ chemotherapy; combin. = combination; PARPi $=$ PARP inhibitor; RIT = radioimmunotherapy. ${ }^{\star \star \star} P<0.001,{ }^{\star \star} P<0.01(2-$ way $\mathrm{ANOVA})$.

bination of EGFR-directed radioimmunotherapy with chemotherapy or PARP inhibitor did not significantly affect the percentage of these cells, the triple-agent combination significantly reduced the percentage of $\mathrm{CD} 44^{+} / \mathrm{CD} 24^{-} /$ $\mathrm{EpCAM}^{+}$in residual tumors.

\section{Treatment of Patient-Derived Grafts and Established Metastases}

As shown in Fig. 5A, the triple-agent combination successfully stopped recurrence of the primary patient-derived TNBC graft (HCI-002) established in the mammary fat pads in a similar manner to the MDA-MB231 xenograft model. Moreover, in a separate model, the triple-agent combination therapy also eliminated experimental metastases. As shown in Fig. 5B, pulmonary metastases of MDA-MB-231 cells were evident by bioluminescence imaging at $14 \mathrm{~d}$ after injection, which became day 0 before combination treatments were administered. Although initial responses were observed in all mice treated with EGFR-directed radioimmunotherapy in combination with PARP inhibitor or chemotherapy, the triple-combination treatment eliminated metastases in $100 \%$ of the mice (Fig. 5B). We did not observe any recurrences in mice (total of 15 mice, 2 independent experiments) that were followed up to $42 \mathrm{~d}$ after treatment with the triple-agent combination.

\section{DISCUSSION}

This preclinical study supported the contention that EGFR-directed radioimmunotherapy in combination with anthracycline/taxane-based chemotherapy and PARP inhibition is effective against EGFR-positive orthotopic and metastatic TNBC models. The combinations of chemotherapy and PARP inhibitors or chemotherapy and anti-EGFR mAbs/inhibitors are currently at the forefront of clinical 


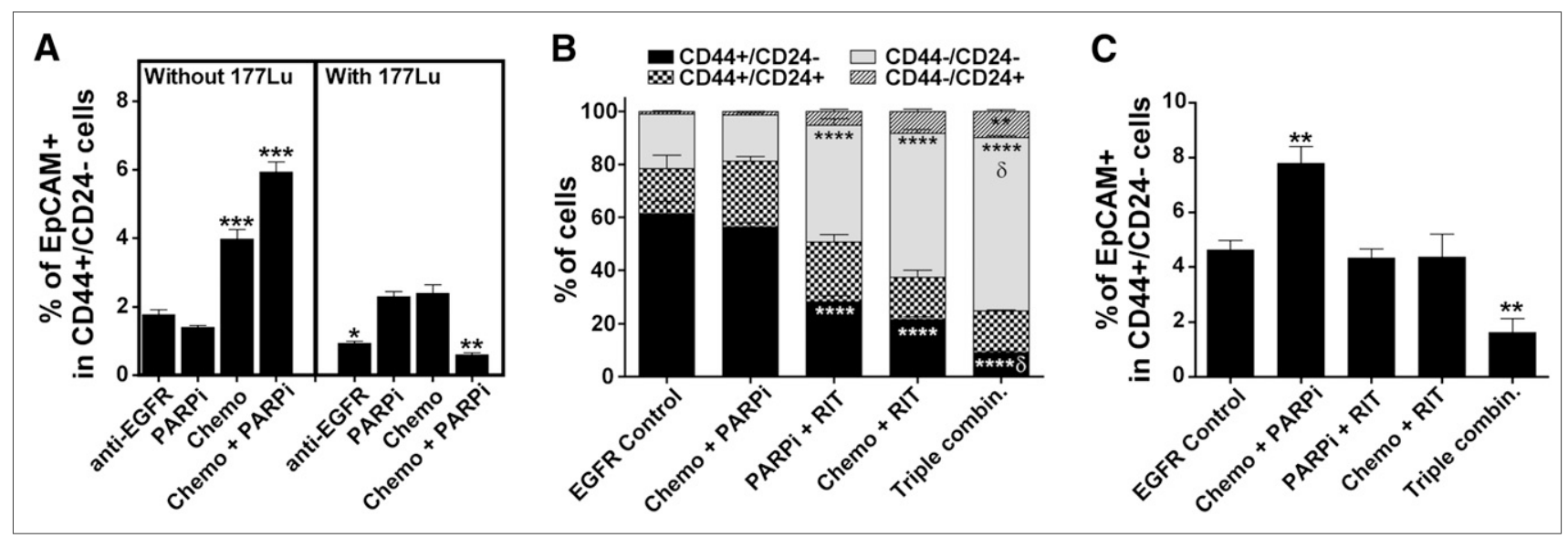

FIGURE 4. In vitro and in vivo demonstration that radioimmunotherapy eliminated BCSCs. (A) Quantification of $B C S C s\left(C D 44^{+} / \mathrm{CD}^{2} 4^{-/}\right.$ $\mathrm{EpCAM}^{+}$) in vitro after various treatments as detected by flow cytometry analysis (3 mice per group). Flow cytometric quantification of $\mathrm{CD} 44 / \mathrm{CD} 24$ (B) and $\mathrm{CD}_{4}{ }^{+} / \mathrm{CD}_{24}{ }^{-} / \mathrm{EpCAM}^{+}$(C) BCSC populations ex vivo using MDA-MB-231 mammary fat pad xenografts isolated on day 10 after treatment of mice (5 mice per group). Supplemental Figure 8 shows representative staining results. Chemo $=$ chemotherapy; combin. = combination; PARPi $=$ PARP inhibitor; RIT = radioimmunotherapy. ${ }^{\star \star \star} P<0.001$, ${ }^{\star \star} P<0.01$ (compared with tumors treated with unlabeled anti-EGFR mAb; 2-way ANOVA). ${ }^{\delta} P<0.01$ (for 3-agent combination compared with 2-agent combinations; 2-way ANOVA).

trials in metastatic breast cancer and $\operatorname{TNBC}(1)$. To our knowledge, this was the first study in which EGFR-directed radioimmunotherapy was used alone or in combination in breast cancer.

The biodistribution and excellent selective tumor targeting of radiolabeled anti-EGFR mAbs in mice are well established (14-16). The uptake of radioimmunoconjugates in the bone (femur), kidneys, and other normal organs was invariably low ( $<5 \%$ injected dose/g), and the highest nontumor uptake was seen in the liver $(\sim 10 \%$ injected dose/g), but this reduced with time. The tumor uptake consistently ranged between $20 \%$ and $40 \%$ injected dose/g and increased with time, showing more than 4-fold tumor-to-liver ratios. ${ }^{90}$ Y-labeled panitumumab showed a significant antitumor effect against head and neck cancer xenografts, with limited toxicity to normal organs (17). On the basis of the tumor uptake in these studies, we can estimate that the dose of ${ }^{177} \mathrm{Lu}$-anti-EGFR $\mathrm{mAb}$ delivered to the tumors ranges between 12 and 25 Gy per gram of tumor within the first $7 \mathrm{~d}$ of treatment. This excellent biodistribution profile explains the lack of toxicity in our experimental groups, the extensive level of cell death in tumors we observed up to $7 \mathrm{~d}$, and the eventual elimination of these tumors, which was associated with the elimination of BCSCs.

The anti-EGFR $225 \mathrm{mAb}$ we used does not bind mouse EGFR, nor does cetuximab or panitumumab. This lack of specificity is described in the literature and was confirmed by us. Unfortunately, a caveat of preclinical models using human tumor xenografts and humanspecific antibodies is that toxicity to normal organs of the murine host may not be accurately determined. Nevertheless, several lines of evidence support the notion that EGFR-targeted radionuclide therapy may be clinically feasible. First, the biodistribution of $\mathrm{mAb}$ against mouse EGFR (7A7) has been investigated in non-tumor-bearing and immunocompetent mice, in which uptake in liver and other normal organs was well below $10 \%$ injected dose/g at $48 \mathrm{~h}$ after injection (18). In a phase I clinical trial performed using the original antiEGFR $225 \mathrm{mAb}$ for safety and imaging (19), the most important observation was the lack of toxicity on the liver, kidneys, or bone marrow in patients who received the 225 $\mathrm{mAb}$ (up to $300 \mathrm{mg}$, including $40 \mathrm{mg}$ of ${ }^{111}$ In-labeled 225 $\mathrm{mAb}$ ). This lack of toxicity was observed in the clinical trials performed in the early 1990s using other anti-EGFR mAbs.

Stronger evidence for the clinical utility of anti-EGFRdirected radioimmunotherapy comes from clinical trials in glioblastoma. In the earliest trial, patients were treated with systemic doses (1.48-5.19 GBq [40-140 mCi]) of ${ }^{131} \mathrm{I}$-antiEGFR mAb (clone EGFR1). With the 3.7-GBq (100-mCi) injection, the estimated dose to tumors was $12.5 \mathrm{~Gy}$ and the estimated dose to bone marrow was $1.4 \mathrm{~Gy}$. No acute toxicities were encountered in any patients, and more important, the authors did not observe any impairment in liver or renal function tests (20). The dose rate from the $\beta$-emission of ${ }^{131} \mathrm{I}(0.10449 \mathrm{~Gy} \cdot \mathrm{g} / \mathrm{MBq} \cdot \mathrm{h})$ is higher than the dose rate of ${ }^{177} \mathrm{Lu}(0.07641 \mathrm{~Gy} \cdot \mathrm{g} / \mathrm{MBq} \cdot \mathrm{h})$. In the second phase II clinical trial, a total of 192 patients with glioblastoma were treated with ${ }^{125} \mathrm{I}$-anti-EGFR mAb (clone 425 ) over a course of 3 weekly intravenous injections of $1.8 \mathrm{GBq}$ (total of 5.4 GBq). No patient required hospitalization or had grade 3 or 4 toxicities with the administration of ${ }^{125}$ I-EGFR mAb (21). EGFR-targeted radioimmunotherapy seems not yet to have been investigated clinically in other cancers, including breast cancer. Encouragingly, a phase I clinical trial (NCT01384253, registered July 2011) of ${ }^{212} \mathrm{~Pb}$-labeled trastuzumab as 


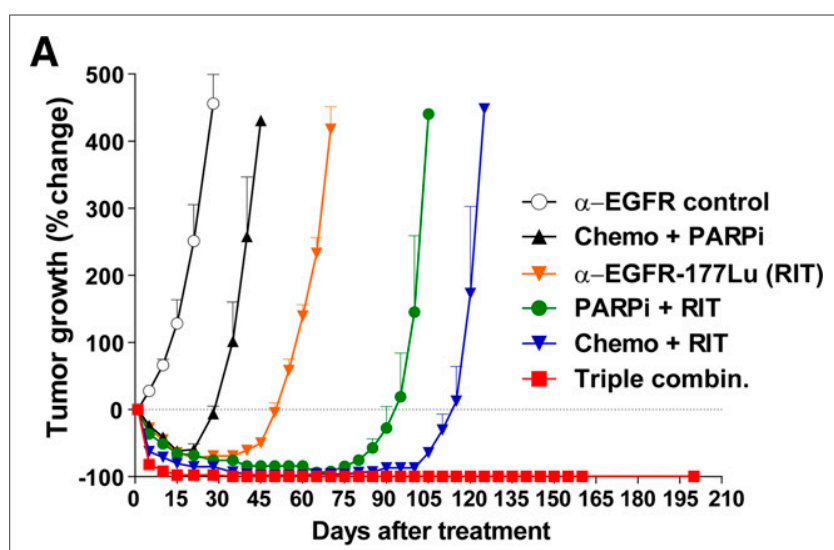

B

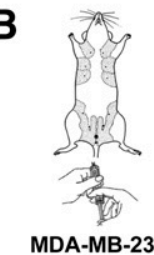

B-231

cells injected

intravenous
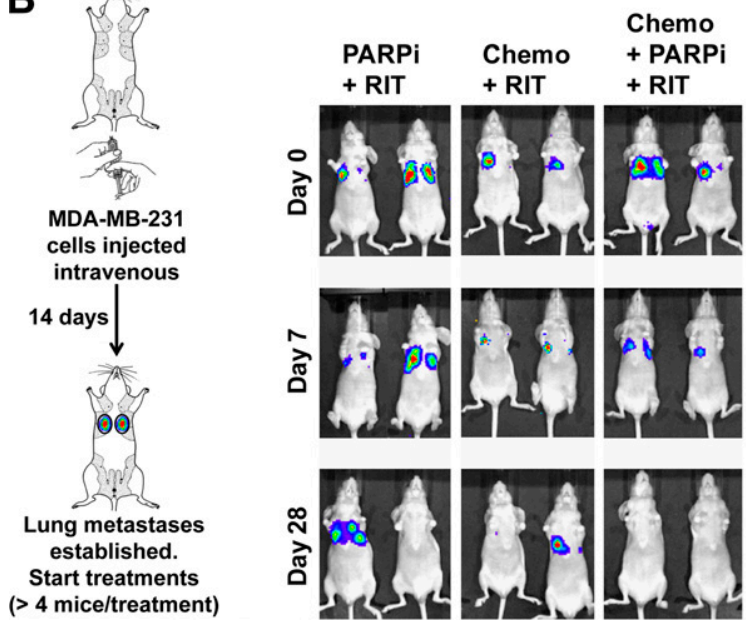

FIGURE 5. Treatment of patient-derived orthotopic grafts and established MDA-MB-231 metastases using triple-combination therapy. (A) Mice bearing patient-derived $\mathrm{HCl}-002$ grafts in mammary fat pads were treated as described in Figure 1 (10 mice per group), and tumors were monitored for growth using caliper measurements. Triple-agent combination stopped recurrences observed in all other treatment arms. (B) In separate model, established metastases (14 d after intravenous injection of MDA-MB-231 cells) were visualized by bioluminescent imaging. Treatments were initiated immediately after imaging (day 0), and mice were imaged weekly thereafter. Combination of EGFR-directed radioimmunotherapy with PARP inhibitor or with chemotherapy showed transient antitumor effect on lung metastases, whereas triple-agent combination eliminated these metastases, with no recurrences observed up to $42 \mathrm{~d}$ after treatment. Only representative images are shown; Supplemental Fig. 8 shows images of all mice.

a targeted $\alpha$-particle anti-HER2 radioimmunotherapy for metastatic disease is recruiting patients with HER2-positive cancers.

There is a strong rationale for targeting EGFR for radioimmunotherapy in TNBC. EGFR is expressed in at least half of TNBC cases, and EGFR has an emerging role in the maintenance of BCSCs, which are associated with disease progression, recurrence, and metastasis (22). The high content of the putative BCSC phenotype, $\mathrm{CD} 44^{+}$/ $\mathrm{CD} 24^{-}$, in tumors from basal-like breast cancer patients correlates positively with elevated EGFR expression $(23,24)$. Functionally, EGFR, HER2, and CD44 form com- plexes in a high percentage of metastatic breast carcinomas (25). This functional interaction regulates shedding, internalization, and motogenic (inducing cellular movement) effects of CD44 in vitro and in vivo and can be inhibited by antibodies against EGFR (26). Inhibition of EGFR signaling, unlike chemotherapy (27), reduces the percentage of $\mathrm{CD} 44^{+} / \mathrm{CD} 24^{-}$cells and disrupts mammosphere formation in cultures (28). Altogether, the literature supports the involvement of EGFR signaling in maintaining BCSCs. However, TNBC cell lines lack sensitivity to EGFR inhibitors (inhibitory concentration of $50 \%$ in the micromolar range) and anti-EGFR antibodies (inhibitory concentration of $50 \%>100 \mu \mathrm{g} / \mathrm{mL}$ ) (29), which has been attributed to pathway activation downstream of EGFR through PI3K/ AKT, loss of PTEN, MEK/ERK/RAS, or localization of EGFR in lipid rafts $(30,31)$. Accordingly, we hypothesized that delivering a therapeutic radionuclide via an anti-EGFR antibody would deplete $\mathrm{CD} 44^{+} / \mathrm{CD} 24^{-}$BCSCs despite downstream compensatory signaling. Indeed, our in vitro data showed that ${ }^{177} \mathrm{Lu}$-anti-EGFR radioimmunotherapy $(10 \mu \mathrm{g} / \mathrm{mL})$ significantly reduced the number of BCSCs when used alone and more so when combined with chemotherapy and PARP inhibitor. Corroborating these findings, the percentage of BCSCs was significantly reduced in tumors when treated with a single dose of ${ }^{177} \mathrm{Lu}$-anti-EGFR radioimmunotherapy. Our data suggest that the high expression of EGFR can be used to target lethal doses of radiation to BCSCs without the need to modulate the EGFR-related canonical signaling networks.

EGFR nuclear translocation, a novel pathway independent of canonical signaling pathways, increases DNA repair after cisplatin and ionizing radiation treatment via increased nuclear retention and activation of DNA-PK (32), which is a critical protein in the nonhomologous end-joining DNA repair pathway. However, EGFR nuclear translocation is inhibited by cetuximab and gefitinib (an EGFR tyrosine kinase inhibitor), which sequester DNA-PK in the cytoplasm thus leading to suppression of DNA-PK activity $(33,34)$. Moreover, EGFR inhibitors decrease RAD51 protein stability and mRNA levels and sequester BRCA1 in the cytoplasm $(35,36)$, which are major players in the homologous recombination DNA repair pathway, thus potentiating the cytotoxicity of chemotherapeutics and radiation $(36,37)$. To this end, we hypothesized that reduced activity of crucial DNA repair proteins would sensitize tumor cells to ${ }^{177} \mathrm{Lu}$-anti-EGFR radioimmunotherapy. In agreement, we found that expression levels of activated DNA-PK (DNAPK S2056) and RAD51 protein were lower in tumors from mice treated with ${ }^{177} \mathrm{Lu}$-anti-EGFR than in tumors from mice treated with chemotherapy and PARP inhibitor. Therefore, incorporating a low dose of radiolabeled anti-EGFR $\mathrm{mAb}$ in the triple-agent combination treatment modulated the direct nuclear EGFR signaling pathway sufficiently to impair DNA repair.

The acknowledged efficacy of combined-modality therapy using external-beam radiotherapy and chemotherapy 
for treating inoperable, locally advanced cancers (38) depends on the radiosensitizing effects of the coadministered cytotoxic drugs (39). Several reports indicate that chemotherapy can sensitize tumors to targeted radionuclide therapy such as radioimmunotherapy (40). In our study, chemotherapy alone significantly potentiated the antitumor effect of ${ }^{177} \mathrm{Lu}$-anti-EGFR radioimmunotherapy. Radiosensitization by PARP inhibition has also been reported previously (39). PARP inhibition in our study potentiated not only chemotherapy but also the antitumor effect of ${ }^{177} \mathrm{Lu}$-anti-EGFR radioimmunotherapy in TNBC models. As far as we know, this is the first report to show that PARP inhibitor augments the effect of low-dose radionuclide therapy.

\section{CONCLUSION}

Our preclinical data support further development of antiEGFR-directed radioimmunotherapy against EGFR-positive TNBC. We observed antitumor activity from a single dose of anti-EGFR-directed radioimmunotherapy alone that was greater than that of the chemotherapy and PARP inhibitor combined. More important, adding anti-EGFR radioimmunotherapy to chemotherapy and PARP inhibitor cured tumor-bearing mice whereas in mice treated with any 2-agent combination, tumors reemerged after initial regression. Mechanistically, tumor relapse in these mice may originate from persistent BCSCs, which in contrast had been eliminated after triple-agent combination treatment. Our results support further investigation of the efficacy of anti-EGFRdirected radioimmunotherapy in rational combinations toward the clinical development of this approach.

\section{DISCLOSURE}

The costs of publication of this article were defrayed in part by the payment of page charges. Therefore, and solely to indicate this fact, this article is hereby marked "advertisement" in accordance with 18 USC section 1734. This work was supported by the Cure Cancer Foundation Australia/Priority-Driven Collaborative Cancer Research Fund (grant 631924), the 2011 Rio Tinto Ride to Conquer Cancer Fund (grant 1017028), and National Health and Medical Research Council (grant 442903). No other potential conflict of interest relevant to this article was reported.

\section{REFERENCES}

1. Reeder-Hayes KE, Carey LA, Sikov WM. Clinical trials in triple negative breast cancer. Breast Dis. 2010;32:123-136.

2. Choi YL, Oh E, Park S, et al. Triple-negative, basal-like, and quintuple-negative breast cancers: better prediction model for survival. BMC Cancer. 2010;10: 507-521.

3. Cheang MC, Voduc D, Bajdik C, et al. Basal-like breast cancer defined by five biomarkers has superior prognostic value than triple-negative phenotype. Clin Cancer Res. 2008;14:1368-1376.

4. Rakha EA, Elsheikh SE, Aleskandarany MA, et al. Triple-negative breast cancer: distinguishing between basal and nonbasal subtypes. Clin Cancer Res. 2009;15: 2302-2310.
5. Liu D, He J, Yuan Z, et al. EGFR expression correlates with decreased diseasefree survival in triple-negative breast cancer: a retrospective analysis based on a tissue microarray. Med Oncol. 2012;29:401-405.

6. Gumuskaya B, Alper M, Hucumenoglu S, Altundag K, Uner A, Guler G. EGFR expression and gene copy number in triple-negative breast carcinoma. Cancer Genet Cytogenet. 2010;203:222-229.

7. Bild AH, Parker JS, Gustafson AM, et al. An integration of complementary strategies for gene-expression analysis to reveal novel therapeutic opportunities for breast cancer. Breast Cancer Res. 2009;11:R55-R64.

8. Hoadley KA, Weigman VJ, Fan C, et al. EGFR associated expression profiles vary with breast tumor subtype. BMC Genomics. 2007;8:258-276.

9. Irshad S, Ashworth A, Tutt A. Therapeutic potential of PARP inhibitors for metastatic breast cancer. Expert Rev Anticancer Ther. 2011;11: 1243-1251.

10. Al-Ejeh F, Darby JM, Tsopelas C, Smyth D, Manavis J, Brown MP. APOMAB, a La-specific monoclonal antibody detects the apoptotic tumor response to lifeprolonging and DNA-damaging chemotherapy. PLOS ONE. 2009;4:e4558e4568.

11. Fillmore CM, Kuperwasser C. Human breast cancer cell lines contain stem-like cells that self-renew, give rise to phenotypically diverse progeny and survive chemotherapy. Breast Cancer Res. 2008;10:R25-R37.

12. Fillmore C, Kuperwasser C. Human breast cancer stem cell markers CD44 and CD24: enriching for cells with functional properties in mice or in man? Breast Cancer Res. 2007;9:303-305.

13. Louderbough JM, Brown JA, Nagle RB, Schroeder JA. CD44 promotes epithelial mammary gland development and exhibits altered localization during cancer progression. Genes Cancer. 2011;2:771-781.

14. Milenic DE, Wong KJ, Baidoo KE, et al. Cetuximab: preclinical evaluation of a monoclonal antibody targeting EGFR for radioimmunodiagnostic and radioimmunotherapeutic applications. Cancer Biother Radiopharm. 2008;23: $619-631$.

15. Nayak TK, Garmestani K, Milenic DE, Baidoo KE, Brechbiel MW. HER1targeted ${ }^{86}$ Y-panitumumab possesses superior targeting characteristics than ${ }^{86}$ Y-cetuximab for PET imaging of human malignant mesothelioma tumors xenografts. PLoS ONE. 2011;6:e18198-e18206.

16. Goldenberg A, Masui H, Divgi C, Kamrath H, Pentlow K, Mendelsohn J. Imaging of human tumor xenografts with an indium-111-labeled anti-epidermal growth factor receptor monoclonal antibody. J Natl Cancer Inst. 1989;81: 1616-1625.

17. Liu Z, Liu Y, Jia B, et al. Epidermal growth factor receptor-targeted radioimmunotherapy of human head and neck cancer xenografts using ${ }^{90} \mathrm{Y}$ labeled fully human antibody panitumumab. Mol Cancer Ther. 2010; 9:2297-2308.

18. Capote AR, González JE, Rodríguez-Vera L, López A, Ramírez BS, Hidalgo GG. Pharmacokinetics and biodistribution study of 7A7 antimouse epidermal growth factor receptor monoclonal antibody and its $\mathrm{F}$ $\left(\mathrm{ab}^{\prime}\right) 2$ fragment in an immunocompetent mouse model. ISRN Pharmacol. In press.

19. Divgi CR, Welt S, Kris M, et al. Phase I and imaging trial of indium 111labeled anti-epidermal growth factor receptor monoclonal antibody 225 in patients with squamous cell lung carcinoma. J Natl Cancer Inst. 1991; 83:97-104.

20. Kalofonos HP, Pawlikowska TR, Hemingway A, et al. Antibody guided diagnosis and therapy of brain gliomas using radiolabeled monoclonal antibodies against epidermal growth factor receptor and placental alkaline phosphatase. $J$ Nucl Med. 1989;30:1636-1645.

21. Li L, Quang TS, Gracely EJ, et al. A phase II study of anti-epidermal growth factor receptor radioimmunotherapy in the treatment of glioblastoma multiforme. J Neurosurg. 2010;113:192-198.

22. Al-Ejeh F, Smart CE, Morrison BJ, et al. Breast cancer stem cells: treatment resistance and therapeutic opportunities. Carcinogenesis. 2011;32: $650-658$.

23. Ricardo S, Vieira AF, Gerhard R, et al. Breast cancer stem cell markers CD44, CD24 and ALDH1: expression distribution within intrinsic molecular subtype. $J$ Clin Pathol. 2011;64:937-946.

24. Honeth G, Bendahl PO, Ringner M, et al. The CD44+/CD24- phenotype is enriched in basal-like breast tumors. Breast Cancer Res. 2008;10: R53-R64.

25. Wobus M, Rangwala R, Sheyn I, et al. CD44 associates with EGFR and erbB2 in metastasizing mammary carcinoma cells. Appl Immunohistochem Mol Morphol. 2002;10:34-39.

26. Pályi-Krekk Z, Barok M, Kovacs T, et al. EGFR and ErbB2 are functionally coupled to CD44 and regulate shedding, internalization and motogenic effect of CD44. Cancer Lett. 2008;263:231-242. 
27. Li X, Lewis MT, Huang J, et al. Intrinsic resistance of tumorigenic breast cancer cells to chemotherapy. J Natl Cancer Inst. 2008;100: $672-679$.

28. Shi Y, Zhang F, Cao M, et al. The role of EGFR MAbs C225 in breast cancer stem cells [abstract]. J Clin Oncol. 2009;27(suppl):e22093.

29. Corkery B, Crown J, Clynes M, O’Donovan N. Epidermal growth factor receptor as a potential therapeutic target in triple-negative breast cancer. Ann Oncol. 2009;20:862-867.

30. Irwin ME, Mueller KL, Bohin N, Ge Y, Boerner JL. Lipid raft localization of EGFR alters the response of cancer cells to the EGFR tyrosine kinase inhibitor gefitinib. J Cell Physiol. 2011;226:2316-2328.

31. de Ruijter TC, Veeck J, de Hoon JP, van Engeland M, Tjan-Heijnen VC. Characteristics of triple-negative breast cancer. J Cancer Res Clin Oncol. 2011;137: 183-192.

32. Liccardi G, Hartley JA, Hochhauser D. EGFR nuclear translocation modulates DNA repair following cisplatin and ionizing radiation treatment. Cancer Res. 2011;71:1103-1114.

33. Dittmann K, Mayer C, Rodemann HP. Inhibition of radiation-induced EGFR nuclear import by C225 (Cetuximab) suppresses DNA-PK activity. Radiother Oncol. 2005;76:157-161.
34. Friedmann BJ, Caplin M, Savic B, et al. Interaction of the epidermal growth factor receptor and the DNA-dependent protein kinase pathway following gefitinib treatment. Mol Cancer Ther. 2006;5:209-218.

35. Ko JC, Hong JH, Wang LH, et al. Role of repair protein Rad51 in regulating the response to gefitinib in human non-small cell lung cancer cells. Mol Cancer Ther. 2008; 7:3632-3641.

36. Ko JC, Ciou SC, Cheng CM, et al. Involvement of Rad51 in cytotoxicity induced by epidermal growth factor receptor inhibitor (gefitinib, IressaR) and chemotherapeutic agents in human lung cancer cells. Carcinogenesis. 2008;29: 1448-1458.

37. Li L, Wang H, Yang ES, Arteaga CL, Xia F. Erlotinib attenuates homologous recombinational repair of chromosomal breaks in human breast cancer cells. Cancer Res. 2008;68:9141-9146.

38. Al-Ejeh F, Brown MP. Combined modality therapy: relevance for targeted radionuclide therapy In: Speer TW, ed. Targeted Radionuclide Therapy. Philadelphia, PA: Lippincott Williams and Wilkins;2010:220-235.

39. Page P, Yang LX. Novel chemoradiosensitizers for cancer therapy. Anticancer Res. 2010;30:3675-3682.

40. Al-Ejeh F, Darby JM, Brown MP. Chemotherapy synergizes with radioimmunotherapy targeting La autoantigen in tumors. PLOS ONE. 2009;4:e4630-e4642. 